\title{
Irritable Bowel Syndrome: Update on Pathogenesis and Management
}

\author{
Osama Alaradi Jamie S. Barkin \\ Division of Gastroenterology, School of Medicine/Mount Sinai Medical Center, University of Miami, \\ Miami, Fla., USA
}

\author{
Key Words \\ Irritable bowel syndrome - Visceral hyperalgesia • \\ Serotonin - Enteric nervous system · Rome criteria • \\ Brain-gut interaction - Motor function · Sensory \\ function - Diarrhea - Constipation
}

\begin{abstract}
Irritable bowel syndrome (IBS) comprises a major proportion of gastrointestinal and primary care practice worldwide. The past several years have seen the rapid evolution of a new and comprehensive model of IBS based on alterations in brain-gut interactions. Alterations in the bidirectional communication between the enteric nervous system and the central nervous system are implicated in the pathogenesis of IBS. 5-Hydroxytryptamine (5-HT; serotonin), a major neurotransmitter in the gastrointestinal tract, and its receptors 5-HT3 and 5-HT4 are involved in the control of gastrointestinal function. A number of abnormal motor and sensory patterns have been reported in patients with IBS. However, it is not known whether these abnormalities are related to symptoms or have a role in establishing a diagnosis of functional gastrointestinal disorders. Visceral hyperalgesia in IBS patients can be secondary to altered receptor sensitivity at the viscus itself and altered central modulation of sensation involving psychological influences in the interpretation of these sensations. The development of diag-
\end{abstract}

nostic criteria for IBS helps to avoid unnecessary and costly investigations. A detailed history allows us to diagnose IBS and search for another cause if warning symptoms are present. The Rome criteria are presently used to define IBS and are currently the most widely applied criteria used in clinical diagnosis and research purposes. Abdominal pain or discomfort associated with chronic altered bowel habits are the mainstay in diagnosis, while the supportive criteria may be used to further classify IBS patients into diarrhea-predominant or constipation-predominant subgroups. Minimal diagnostic tests have been advocated in the initial diagnostic approach to patients with suspected IBS, depending on the predominant symptom. The therapeutic goals in IBS must focus on the overall well-being of the patient, including abdominal symptoms and the accompanying nonbowel symptoms and affective disorders. It is important to establish an effective physician-patient relationship and to reassure the patient once the diagnosis of IBS is made. Dietary modification may be of value in some patients with IBS. Dietary fiber is frequently recommended for patients with constipation-predominant IBS. Two novel serotonin agonists are currently under development for constipated IBS patients, tegaserod and prucalopride. Antidiarrheal agents, including loperamide and diphenoxylate, may help patients with diarrhea-predominant IBS. 5-HT3 receptor antagonists may play a role in the management of such patients in the future.

\begin{tabular}{ll}
\hline KARGER & ( ) 2002 S. Karger AG, Basel \\
Fax +4161306 1234 & 1011-7571/02/0111-0002\$18.50/0 \\
$\begin{array}{l}\text { E-Mail karger@karger.ch } \\
\text { www.karger.com }\end{array}$ & $\begin{array}{l}\text { Accessible online at: } \\
\text { www.karger.com/journals/mpp }\end{array}$
\end{tabular}

Jamie S. Barkin, MD

Division of Gastroenterology

Mount Sinai Medical Center, 4300 Alton Road

Miami Beach, FL 33140 (USA)

Tel. +1 305674 2240, Fax +1 305674 2671, E-Mail s-vance@msmc.com 
Psychological treatment and antidepressants should be considered when IBS symptoms are severe or refractory or associated with psychological distress and impaired quality of life.

Copyright $@ 2002$ S. Karger AG, Basel

\section{Introduction}

Irritable bowel syndrome (IBS) comprises a major proportion of gastrointestinal and primary care practice worldwide. It affects between 9 and $22 \%$ of the population in the United States and 3.5-25\% worldwide. Moreover, it produces considerable costs to society in terms of lost work, consultation, unnecessary tests and inappropriate management [1]. Over the past two decades, there has been a remarkable increase in research that has led to a better understanding of this illness, including the interaction between the gastrointestinal tract and the nervous system. IBS and functional gastrointestinal disorders are now viewed as clinical manifestations of alterations in the bidirectional communication between the gastrointestinal tract and the nervous system [2]. Dysregulation of intestinal motor, sensory and central nervous system function contributes to symptoms in IBS patients [3]. The symptoms may be modified by sociocultural and psychosocial influences and in the absence of a reproducible and accepted biological marker, symptoms currently remain the primary means of diagnosing patients [4]. During the past decade, there has been a remarkable growth in investigative techniques that support this new concept of braingut interaction, including imaging of the brain (positron emission tomography and functional magnetic resonance imaging), improved motility assessment and the molecular investigation of brain-gut peptides [5]. As a result, several biological therapeutic targets have been identified, resulting in the development of new therapeutic drugs for IBS [6]. We will review the recent advances in the pathophysiology of IBS and the clinical approach to the management of patients with IBS.

\section{Epidemiology}

Several epidemiological studies in the USA have indicated that the prevalence of IBS ranges between 9 and $22 \%$ [7-10]. This is similar to its worldwide prevalence, which ranges between 3.5 and 25\% [11]. Although there are very few data that address the prevalence of IBS in non-Western countries, the available studies suggest that the prevalence of IBS in Japan, China and parts of Africa is similar to that in the United States [12-14]. The prevalence estimates vary because of the diversity of definitional criteria and differences in the specific questions used to elicit the information.

In Britain and the USA, the available data indicate that IBS affects $14-24 \%$ of women and 5-19\% of men. The ratio of females to males is $2: 1$ within those who have symptoms but do not seek medical care and 3:1 within the patient population who seek consultation with their primary care physicians. The prevalence seems similar in whites and blacks, but lower in Hispanics and elderly patients [3].

It is estimated that only $10 \%$ of people with IBS symptoms seek medical care for evaluation of their symptoms [15]. Nevertheless, IBS accounts for 3.5 million physician visits in the USA annually and is the most common diagnosis in gastroenterology practice - about $25 \%$ of all patients [16, 17]. IBS accounts for $12 \%$ of primary care, and a survey of American Gastrointestinal Association members indicated that IBS comprised $28 \%$ of all gastroenterology practice and all functional gastrointestinal disorders $41 \%$ [18]. Its economic impact is enormous. At least 8 billion dollars are spent annually on health service charges for subjects with IBS [19]. This is probably grossly underestimated, since neither outpatient drug costs and indirect costs were measured [20], nor the costs to society.

IBS is a major cause of absenteeism from work as well, resulting in decreased productivity. These factors contribute to the indirect costs, which are probably much greater than the direct costs. The US Householder Survey estimated that the adjusted mean number of days of work absenteeism over a 1-year period was 13.4 days for those with IBS compared with a mean of 4.9 days for persons without functional gastrointestinal complaints [7]. In addition, persons affected with IBS pass up promotions compared with their counterparts.

\section{Pathophysiology}

The past several years have seen the rapid evolution of a new and comprehensive disease model based on alterations in brain-gut interactions [21]. Research in neurogastroenterology has led to a better understanding of the arrangement, function and malfunction of the enteric division of the autonomic innervation of the digestive tract and its relationship to sympathetic and parasympathetic systems, as well as to the spinal cord and the brain 
[22]. Many of the symptoms characteristic of functional gastrointestinal disorders are consistent with dysfunction of the motor and/or sensory apparatus of the digestive tract [23]. Aspects of sensorimotor dysfunction most relevant to functional gastrointestinal disorders include alteration of gut contractile activity, myoelectrical activity and transit, as well as enhanced perceptual responses to visceral stimuli in different regions of the gastrointestinal tract [23].

\section{Brain-Gut Interaction}

Understanding the basic normal structural arrangement of the enteric nervous system is important in understanding the pathophysiology of IBS. The gastrointestinal tract is supplied by the enteric nervous system, which has local circuits for integrative functions independent of extrinsic nervous connections. It is connected to the central nervous system through the sympathetic and parasympathetic systems. Final common pathways for output from higher centers to the gut exit the brain in afferent vagal fibers and descending pathways in the spinal cord that connect to sympathetic and parasympathetic preganglionic neurons [22].

Several brain centers, including areas in the frontal lobe, hypothalamus and amygdala nucleus, project to the vagal outflow tract in the medulla oblongata. These areas share information with the limbic system, where emotional responses to sensory output from the outside world are processed. Interactions between higher brain centers, emotional state and gastrointestinal disorders are well recognized. Abdominal pain, diarrhea, nausea and vomiting can all be manifestations of emotional or traumatic stress [22]. Evidence from ultrastructural and light microscopic studies suggests that the enteric mast cells are innervated by projections of the central nervous system. In animal studies, stimulation of these cells by thyrotropin-releasing hormone evokes degranulation of mucosal mast cells in the small intestine [24]. In the large bowel of rats, restraint stress exacerbates nociceptor responses, and these effects are associated with increased release of histamine [25]. Mast cell degranulation may release mediators that sensitize silent nociceptors in the large intestine. In animal studies, degranulation of intestinal mast cells results in a reduced threshold for the pain response to balloon distension [26]. The brain-to-mast cell connection appears to be a mechanism that can link the psychoemotional status to the irritable state of the gastrointestinal tract. The irritable state of the bowel (abdominal discomfort and diarrhea) known to result from degranulation of intestinal mast cells and release of signals to the enteric nervous sys- tem is expected to occur irrespective of the mode of stimulation of mast cells [22].

Inflammation of the mucosa of the gastrointestinal tract, accompanied by changes in the enteric nervous system and smooth muscle function leading to sensorimotor abnormalities, has been proposed as one of the mechanisms producing IBS symptoms. This hypothesis is supported by clinical observations and studies showing a higher incidence of IBS following enteric infections compared to the general population. In a cohort study, patients with a bacteriologically confirmed episode of gastroenteritis were 10 times more likely to have IBS than were subjects in the general population [27]. However, the criteria used for diagnosing IBS were not mentioned in the study. Gwee et al. [28] have shown that about a quarter of patients with acute gastroenteritis subsequently developed IBS symptoms. At the time of their illness, these patients had higher scores for anxiety, depression, somatization and neuroticism. The predictive value of stressful life events and hypochondriasis measures was highly significant and independent of other psychological factors [28]. In those patients who had rectal biopsy 3-6 months after gastroenteritis, chronic inflammatory cells were significantly higher in patients who developed IBS compared with controls and those who did not develop IBS. A postulated mechanism of postinfectious IBS is that in psychologically susceptible individuals, enteric infections cause neuroimmune interactions which result in continuing sensorimotor dysfunction. Although the role of infection and stress in the pathogenesis of IBS is still controversial, the data are provocative and certainly well designed longer-term studies are needed to delineate the relation better.

The enteric nervous system, a subdivision of the autonomic nervous system, has bidirectional communication with the central nervous system. It is considered a local minibrain, within which is stored a library of programs for different patterns of gut behavior [22]. The enteric nervous system includes sensory, motor and interneurons. Sensory neurons have receptors for detecting changes in thermal, chemical or mechanical stimulus energy. Interneurons are connected by synapses into networks that process sensory information and control the behavior of motor function. Motor neurons are the final common pathway for the transmission of control signals to the effector system. A major neurotransmitter in the gastrointestinal tract is 5-hydroxytryptamine (5-HT; serotonin), which is found in enterochromaffin cells located in the intestinal epithelium, in interneurons terminating in the myenteric and submucous plexuses and in mast cells 
[6]. The role of 5-HT in normal and pathological gastrointestinal conditions is apparent in its pattern of distribution in the body. Around 95\% of 5-HT in the body is in the gastrointestinal tract and about 5\% is in the brain [29]. Serotonin is present in enteric nerves and is synthesized in the enteric nervous system. Serotonergic neurons constitute about $2 \%$ of all myenteric neurons. Serotonin is released from the bowel when the enteric nerves are stimulated. It has diverse motor and sensory functions in the gastrointestinal tract through submucosal and myenteric neurons that respond to 5-HT through a variety of receptors. 5-HT receptors are present on enteric neurons, enterochromaffin cells, gastrointestinal smooth muscle cells and possibly on enterocytes and immune tissue [30]. The various effects of 5-HT in the gut and the brain are attributable to the existence of as many as 14 receptor subtypes that are known to date. While the 5-HT1, 5-HT2, 5-HT3 and 5-HT4 receptor subtypes predominate in the gastrointestinal tract, it is the 5-HT3 and 5-HT4 receptors, involved in the control of gastrointestinal function, that have been implicated in the pathophysiology of IBS. Intraluminal stimulation facilitates the release of 5-HT, which activates the peristaltic reflex [31]. The release of 5-HT, triggered by intraluminal movement of a bolus, activates intrinsic primary afferent neurons that synapse with interneurons in the submucosal plexus or the myenteric plexus. A bifurcating signal transduction cascade occurs, facilitating smooth muscle contraction on the proximal end of the bolus and, simultaneously, the relaxation of muscles on the distal end, thereby propelling the bolus unidirectionally. The 5-HT4 receptors located in cholinergic and motor neurons are important mediators of this peristaltic reflex, as evidenced by an in vitro study of tegaserod, which is a 5-HT4 receptor agonist [32-34]. 5-HT3 receptors are widely distributed on the enteric neurons, particularly substance P-containing afferent neurons that are involved in local reflexes within the gut $[32,35]$. There is evidence to suggest that 5-HT3 receptor antagonists modulate visceral pain pathways in humans [35]. A potential mechanism of action is that 5-HT3 antagonists decrease visceral nociception by selectively blocking 5-HT3 receptors on visceral afferent neurons.

\section{Abnormal Motor Function}

A number of abnormal motor and sensory patterns have been reported in some patients with IBS $[11,21,36]$. However, it is not known whether these abnormalities are related to symptoms or have a role in establishing a diagnosis of functional gastrointestinal disorders [23]. Most studies have been undertaken to evaluate the colon and the rectum because of their greater accessibility [23]. Alterations in colorectal motility include increased postprandial colonic phase contractions in patients with IBS with a clinically prominent gastrocolonic reflex, increased colonic contractions in IBS patients with predominant constipation, reduced contractions in patients with diarrhea-predominant IBS and impaired adaptive relaxation of the rectum in response to chronic distension in IBS patients [23].

The colon of patients with diarrhea-predominant IBS demonstrates a greater number of fast contractions and propagated contractions [37]. In contrast, patients with constipation-predominant IBS have fewer high-amplitude propagated contractions [38]. Accelerated transit of a meal through the colon was found in patients with diarrhea-predominant IBS, and delayed transit was found in patients with constipation-predominant IBS [39]. In some studies, an increase in perception of the apparently normal events in the gut was found in a subgroup of patients with IBS [23]. Although there are a few reports describing minor abnormalities in rectal and colonic compliance, generally it remains normal overall in IBS patients [23]. An exaggerated sigmoid response to systemic and psychological stresses is likely to be related to enhanced output from parasympathetic neurons receiving input from pontine Barrington's nucleus [40, 41]. Based on current evidence, it is plausible to assume that alterations in autonomic regulation of the small and large intestine play an important role in the generation of altered bowel habits. It remains to be determined how important these autonomic alterations and associated motility changes are in the generation of symptoms in IBS patients.

\section{Abnormal Sensory Function}

Most stimuli in the gut are not consciously perceived. Hypersensitivity is the excessive perception of gut stimuli or an excessive afferent response to stimuli [23]. A proportion of patients with IBS display an increased conscious perception of painful distension in the small bowel and colon, and in some studies, an increased perception of apparently normal motor events in the gut has been found [42]. Abnormal visceral perception is suggested by excessive sensitivity to balloon distension documented at the rectosigmoid and the anorectum [43, 44]. Diarrhea-predominant IBS patients exhibit lower thresholds for the sensation of gas, stool and discomfort in the anorectum, and urgency and discomfort develops at lower volumes of distension of an intrarectal balloon. In contrast, patients with constipation-predominant IBS develop discomfort at greater distension volumes than healthy controls [43- 
45]. The consistency with which patients with IBS are found to have lower pain thresholds with balloon inflation led Mertz et al. [46] to speculate that rectal pain sensitivity is a biological marker for IBS and that this biological marker reflects alterations in stretch receptors or spinal pathways for pain. However, a retrospective review of the literature relating to pain thresholds in patients with IBS between 1973 and 1997 concluded that visceral hyperalgesia cannot be explained on the basis of biological differences between patients with IBS and controls, and that psychological factors influence the perception of pain [47]. This is consistent with the emerging view of IBS as a more integrated, biopsychosocial model of illness. Although the search continues to find a biological marker to measure symptoms and monitor the response to treatment, such a marker has not yet been found. Patients with IBS also display a distorted referral pattern of gut sensation and perceive intestinal distension more diffusely over the abdomen than healthy controls [48].

Normally, visceral pain information travels through Adelta and $\mathrm{C}$ fibers and synapses in the dorsal horn of the spinal cord, specifically laminae I and II. The neural signal ascends through the contralateral spinothalamic tract to the brain, where pain is perceived. Several mechanisms at different sites of the brain-gut interconnections have been proposed to explain visceral hyperalgesia in IBS patients. These include altered receptor sensitivity at the viscus itself, increased excitability of the spinal cord dorsal horn neurons, where repeated distension of the intestine produces spinal hyperalgesia with somatic referral of the visceral stimulus, and altered central modulation of sensation involving psychological influences in the interpretation of these sensations [23, 45]. Several substances may influence sensation by acting at different levels of the brain-gut connection. The specific 5-HT3 receptor antagonists such as ondansetron and the somatostatin analogue octreotide may have peripheral antinociceptive activity and have been shown to modulate visceral pain pathways in humans [23, 35]. Studies in rats have demonstrated that noxious colorectal distension increases the expression of the proto-oncogene c-fos in laminae I and II (spinal cord pain center) of the lumbosacral dorsal horn. Pretreatment with the 5-HT3 receptor antagonist alosetron significantly reduced the c-fos expression in laminae I and II in a dose-dependent manner, indicating a role of 5-HT3 receptors in the spinal processing of visceral nociception [49]. Somatostatin immunoreactivity is found in dorsal root ganglia and nerves in the dorsal horn, and there is evidence to suggest that the endogenous release of somatostatin modulates neuronal transmission in the spinal cord by depressing the excitability of the dorsal horn neurons. Opioid agonists of the mu, alpha and kappa receptors all have antinociceptive effects in the gut [50]. Kappa receptor agonists like fedotozine, which reduce visceral hypersensitivity and affect the sensory threshold to gastric and colonic distension in humans, have been shown to improve symptoms in IBS [51].

Higher neural centers play a role in the interpretation and modulation of the sensory signals ascending from the gastrointestinal tract. The mechanisms of central interpretation of afferent signals are not known. In one study, the relationship between visceral pain perception and regional cerebral activity was evaluated in normal subjects and patients with IBS using positron emission tomography and ${ }^{15} \mathrm{O}$-water, a radiotracer that reflects by its spatial distribution the pattern of regional cerebral blood flow at the time of its transit through the brain [52]. A total of 12 patients ( 6 with IBS and 6 healthy) were studied during nonpainful, painful and stimulated rectal balloon pressure pulses. It was found that the perception of acute rectal pain is associated with activation of the anterior cingulate gyrus in healthy subjects, while patients with IBS show an aberrant brain activation pattern in the prefrontal cortex both during noxious rectal distension and during the anticipation of rectal pain [52]. Linked interactions between higher brain centers, emotional state and gastrointestinal disorders are clinically well recognized, as abdominal pain, diarrhea and vomiting can all be manifestations of emotional or traumatic stress. The role of the central nervous system in modulating IBS symptoms is supported by evidence that during sleep, motility disturbances disappear, while with alertness and arousal, the propagating velocity increases progressively and the frequency of migrating motor responses decreases.

Patients with IBS have a variety of extraintestinal symptoms, including fibromyalgia, irritable urinary bladder and alterations in vital functions such as sleep, libido, appetite and energy level [53]. Some of these symptoms can be explained by alterations in the interactions between the nervous system, the viscera and the musculoskeletal system. Under normal conditions, the great majority of visceral afferent innervation is not consciously perceived. Unlike somatosensory innervation, only a small percentage of afferent fibers innervate the viscera, and thus a highly developed afferent signaling system from the viscera to the brain is lacking [53]. Visceral afferent fibers converge onto dorsal horn neurons within the spinal cord that primarily receive input from somatic structures. Fibers from a particular organ show highly 
divergent projections to the spinal cord, often to more than 6 spinal segments [54]. As a result of this divergence, there is considerable convergence of afferent input from different viscera and somatosensory systems. Dorsal horn neurons become more excitable in response to increased afferent input from the periphery or in response to changes in descending pain modulation systems. Subthreshold input from viscera is then able to excite dorsal horn neurons [21,26].

\section{Psychosocial Factors}

The link between psychological factors and functional gut disorders is based on (1) epidemiologic studies, (2) the relationship between life events and gastrointestinal symptoms, (3) physiological studies demonstrating changes related to stress and the response of some of these disorders to drugs which modify psychological well-being, and lastly (4) psychological interventions such as counseling and behavioral therapy, which can modify symptoms of IBS [55]. Psychological factors have no diagnostic value for IBS and are not included in the diagnostic criteria. However, the identification of specific psychological stressors associated with the exacerbation of symptoms may help in planning treatment through psychological or psychopharmacological interventions [3]. Studies have shown that patients with IBS often report that stressful events precede the onset or exacerbation of their symptoms. Whitehead et al. [56], using a life event questionnaire given every 3 months for 1 year, showed that IBS subjects had significantly higher levels of stress than those with other functional bowel disorders or those in control groups. Creed et al. [57] found that a group of subjects with functional abdominal pain and symptom onset of less than 12 months had experienced several life events significantly greater than either the organic or healthy comparison groups. These events included separation from a spouse, divorce, court appearance with threat of imprisonment or deaths in the family [57]. In a questionnaire study of 135 patients with IBS and 654 controls, $73 \%$ of the IBS patients and $54 \%$ of the control group reported that stress altered their stool pattern, whereas $84 \%$ of the IBS patients and $68 \%$ of the control group reported that stress led to abdominal pain [58]. Up to $60 \%$ of patients with IBS have been reported to have a psychiatric disorder, which is considerably higher than the rate of psychiatric disorders in patients with organic disease or healthy controls [59]. Patients with IBS who had not consulted a physician had the same rate of psychiatric disorders as the general community [59, 60]. However, patients with IBS who consulted a physician had signifi-
Table 1. The Manning criteria [64]

$$
\begin{aligned}
& \text { Pain relieved by defecation } \\
& \text { Looser stools at onset of pain } \\
& \text { More frequent stools at onset of pain } \\
& \text { Visible abdominal distension } \\
& \text { Passage of mucus } \\
& \text { Sensation of incomplete evacuation }
\end{aligned}
$$

cantly more psychological symptoms than asymptomatic controls or nonconsulters [60]. Psychological disorders in this study included depression, anxiety, somatization, paranoia and obsessive-compulsive phenomena.

Comorbid psychiatric diagnoses (the most common being panic and generalized anxiety disorder), mood disorders (major depression and dysthymic disorder) and somatoform disorders (hypochondriasis and somatization disorder) are present in up to $42-61 \%$ of patients with IBS compared to $19 \%$ of patients with organic gastrointestinal disorders and 16\% of healthy controls [61]. There are also reports of high frequency rates (30-56\%) of sexual and physical abuse among IBS patients [62]. Patients with IBS and functional dyspepsia have impaired quality of life that is more severe than the impairment in patients with structural abnormalities such as peptic ulcer and liver disease [63].

\section{Diagnostic Criteria}

It is important to obtain a detailed history and identify warning symptoms which may suggest organic disease like rectal bleeding, loss of weight and appetite and family history of colon cancer and inflammatory bowel disease. Use of the available diagnostic criteria and basic laboratory testing circumvent unnecessary and costly investigations in patients with suspected IBS. Manning et al. [64] were the first to suggest specific diagnostic criteria for the diagnosis of IBS (table 1). Four symptoms were identified as being significantly more common in patients with IBS. These were relief of abdominal pain with bowel movement, more frequent stools with the onset of pain, loose stools with the onset of pain and visible abdominal distension [64]. In the same study, there was a trend for increased passage of mucus and a feeling of incomplete rectal evacuation in IBS patients. In subsequent studies from Canada and the USA, it has been confirmed that the greater the number of these 6 symptom criteria that are present, the greater the probability that the diagnosis is IBS. This is specially so in young patients and in females $[65,66]$. Studies that evaluated the Manning criteria 
Table 2. Rome criteria for IBS

a Rome I criteria for IBS [74]

Continuous or recurrent symptoms of

1 abdominal pain or discomfort that is relieved with defecation, and/or

associated with a change in frequency or form of stool, and/or

associated with a change in consistency of stool, and

2 two or more of the following, on at least one quarter of occasions or days:

altered stool frequency (for research purposes, altered may be defined as $>3$ bowel movements per day or $<3$ bowel movements per week)

altered stool form (lumpy/hard or loose/watery stool)

altered stool passage (straining, urgency or feeling of incomplete evacuation)

passage of mucus

bloating or feeling of abdominal distension

b Rome II criteria for IBS [75]

At least 12 weeks, which need not be consecutive, in the preceding 12 months of abdominal discomfort or pain that has two of three features:

1 relieved with defecation, and/or

2 onset associated with a change in frequency of stool, and/or

3 onset associated with a change in form (appearance) of stool

Supportive symptoms of IBS:

1 fewer than 3 bowel movements a week

2 more than 3 bowel movements a day

3 hard or lumpy stools

4 loose (mushy) or watery stools

5 straining during a bowel movement

6 urgency (having to rush to have a bowel movement)

7 feeling of incomplete bowel movement

8 passing mucus (white material) during a bowel movement

9 abdominal fullness, bloating or swelling

Diarrhea-predominant IBS: one or more of 2, 4 or 6 and none of 1,3 or 5

Constipation-predominant IBS:

one or more of 1,3 or 5 and none of 2,4 or 6

showed they have a specificity ranging between 53 and $86 \%$ and a positive predictive value of $48-83 \%$ [65-69].

Another approach to distinguish IBS from organic disease was developed in 1984 by Kruis et al. [70]. This diagnostic scoring system included: (1) the presence of abdominal pain, flatulence and bowel irregularity, (2) symp- toms for more than 2 years, (3) the description of pain, (4) alternating constipation and diarrhea, (5) abnormal physical findings, (6) an erythrocyte sedimentation rate greater than $10 \mathrm{~mm} / \mathrm{h},(7)$ a white cell count greater than $10,000 / \mathrm{mm}^{3}$ and (8) anemia [70]. The last four criteria include physical signs and laboratory findings, the presence of which is suggestive of organic disease. This is different from the Manning and the Rome criteria, which depend entirely on symptoms. Using this score, the investigators concluded that a detailed history, physical examination and basic laboratory tests are sufficient in most patients for the positive diagnosis of IBS and the exclusion of any underlying organic disease [70]. However, the tests carried much greater weight than symptom items in this scoring system. Studies evaluating the diagnostic value of the Kruis scoring system showed variable results as well $[71,72]$. In one study three or more of the Manning criteria discriminated IBS from organic disease among 347 outpatients with a sensitivity of $90 \%$ and a specificity of $87 \%$, while the Manning and the Kruis criteria combined had a sensitivity of $80 \%$ and a specificity of $97 \%$ [73].

The Rome criteria are based on the gastrointestinal symptoms described by both Manning and Kruis [4]. These are presently used to define IBS and are currently the most widely applied criteria used in clinical diagnosis and for research purposes [1,2]. They were developed at a consensus meeting in Rome in 1988 (Rome I) (table 2a) and were revised in 1998 (Rome II) (table 2b) [4, 5, 74, 75]. The Rome I criteria recommend the diagnosis of IBS only in the presence of the main diagnostic criteria; that is, abdominal pain or discomfort associated with chronic altered bowel habits and two or more of the supportive criteria. The Rome II criteria recommend that the diagnosis of IBS be based on the presence of abdominal pain plus two of the three main diagnostic criteria. The supportive criteria may then be used to further classify IBS into diarrhea-predominant or constipation-predominant subgroups $[4,75]$. The duration of symptoms in the Rome II criteria was specified as 12 weeks (not necessarily consecutive) during the preceding 12 months in the absence of a structural or biochemical explanation [5]. It should be clear that the Rome criteria emphasize a positive diagnosis rather than excluding other diseases. The validity of the Rome criteria in discriminating IBS from organic disease has not been studied. Several studies have evaluated the validity of the symptom-based diagnostic criteria but most of them had potential problems and only a few were true population studies [4]. A recent population-based study which used both factor and cluster analysis suggested that distinct groupings of the symptoms and subjects 
do exist in the population, and IBS defined by the Rome criteria is one of the clusters that can be defined [76]. Vanner et al. [77], by evaluating 95 patients prospectively and 98 patients retrospectively, showed that the Rome criteria and the absence of red flags (table 3 ) had a sensitivity of $65 \%$, a specificity of $100 \%$ and a positive predictive value of $100 \%$ in the retrospective group and a positive predictive value of $98 \%$ in the prospective group. The American Gastroenterological Association's technical review for developing practice guidelines for IBS recommended a sequential diagnostic strategy that includes the use of symptom-based criteria (Rome criteria), a conservative evaluation strategy based on the predominant symptom(s) and initiation of symptomatic treatment with reassessment in several weeks [3].

Patients who only have chronic abdominal pain are no longer classified as having IBS; rather, these patients are considered to have functional abdominal pain. Similarly, patients with chronic unexplained diarrhea and no abdominal pain are no longer considered to fall within the spectrum of IBS $[71,75]$. There are a few potential limitations to the Rome criteria. Postprandial symptoms such as abdominal pain or urgency, which may represent an exaggerated gastrocolonic response in IBS patients, were not included, and in our opinion, should be included. Moreover, the diagnostic value of the Rome criteria in men may be lower than it is in women. Another contentious issue is whether it is too restrictive to insist on abdominal pain being present [71]. Despite the limitations of the current symptom-based criteria, however, there remains consensus that a positive diagnosis of IBS can be made based on the symptom pattern in the published criteria and limited investigations to exclude organic diseases that have a similar presentation [3].

\section{Symptoms}

The symptoms of IBS often overlap with those of other functional gastrointestinal disorders. The US Householder Survey of functional gastrointestinal disorders, for example, showed that in patients whose bowel symptoms included IBS, functional constipation and functional diarrhea, esophageal symptoms were reported by $56 \%$, gastroduodenal symptoms by $37 \%$ and anorectal symptoms by $41 \%$ [3]. Extraintestinal symptoms are also common in patients with IBS [53, 78]. Talley et al. [79] reported that extraintestinal symptoms, which included headache, backache, insomnia, fatigue, weakness, stiffness, dizziness and palpitations, were more common in functional and organic disease groups compared to healthy controls. However, these symptoms were not useful in discriminat-
Table 3. Red flags [2]

\author{
Documented weight loss \\ Nocturnal symptoms \\ Blood mixed in the stools \\ Recent antibiotic use \\ Family history of colon cancer \\ Relevant abnormalities on physical \\ examination
}

ing functional from organic gastrointestinal disease [79]. Other extraintestinal symptoms that are associated with IBS include dyspareunia, dysuria, nocturia, urinary urgency, lethargy, headache and muscle and back pain [53, 78]. There is also much overlap of IBS with other functional disorders such as fibromyalgia, premenstrual syndrome, noncardiac chest pain, chronic fatigue syndrome, hyperventilation syndrome, tension headache, atypical facial pain, globus syndrome and multiple chemical sensitivity. Wessely et al. [80] in a review of these functional syndromes, concluded that there are apparent similarities in case definition, reported symptoms and nonsymptom association which include factors such as the patient's sex and response to treatment. Their review confirms that patients who meet the criteria for a specific functional syndrome report symptoms that define other syndromes. This overlap implies the consideration of classifying these syndromes as variants of a general functional somatic syndrome and that a more general broad-based approach aided by liaison with psychiatrists or psychologists should be taken. These findings should encourage us to look for other comorbid syndromes in our IBS patients and manage the patient as a whole.

A careful interpretation of the pain and stool characteristics is the most important step in raising the clinical suspicion of IBS [1]. The current Rome criteria require that abdominal pain and disturbed defecation must both be present for the diagnosis to be made $[4,75]$. The criteria do not give a specific definition of the abdominal pain or discomfort and thus are subject to individual interpretation [4]. Pain may become more severe with increasing duration and severity of constipation. Frequently, there is a sense of incomplete evacuation leading to repeated attempts at defecation. Relief of the abdominal pain by defecation is one of the key items identified by Manning et al. [64]. However, any colonic pain may be relieved by defecation. Indeed, in inflammatory bowel disease, this occurs as frequently as it does in IBS [60]. The association of the abdominal pain with a change in the frequency or 
consistency of stools is important in both sets of IBS criteria $[64,81,82]$. The frequency and quality of each symptom, although highly variable between patients, are fairly consistent for an individual patient. Patients with constipation-predominant IBS may have many days of constipation interrupted with brief episodes of diarrhea. The constipation may be continuous and increasingly intractable to laxatives and enemas, and the stools may be hard, pellet-like or pencil-like in caliber. The diarrhea in IBS usually consists of a small volume of stools which may be described as loose, mushy or watery. Bowel movements may follow meals and can be explosive. Typically, they are absent during sleep.

The differential diagnosis of IBS includes lactose intolerance, gastrointestinal infections, inflammatory bowel disease, thyroid disease, endocrine tumors, colorectal cancer, microscopic colitis and malabsorption syndromes $[60,81]$. It is important to keep in mind that by statistical coincidence, a common condition such as IBS, which affects up to $20 \%$ of the population, can coexist with organic disease [1]. Thus, we need to search for a superimposed problem in our IBS patients with unusual exacerbations.

\section{Predominant Symptom-Based Diagnostic Approach}

It may be important for practicing physicians to divide patients with IBS into subgroups in order to optimize their treatment responses, as well as to evaluate the outcome of clinical trials that involve novel therapeutic modalities [83]. Recent studies have reported pathophysiologic differences in the reporting and perceptual responses among these subgroups and support a symptombased diagnostic approach [1]. IBS patients with constipation predominance, as expected, mainly complain of infrequent bowel movements, hard or lumpy stools, a sensation of incomplete evacuation or excessive straining with no loose or watery stools [1,2]. Compared to patients with diarrhea-predominant IBS, those with constipationpredominant IBS more frequently report musculoskeletal symptoms, dyspepsia-associated symptoms and alteration in vital functions such as poor sleep, loss of appetite and sexual dysfunction [1]. Patients with diarrhea-predominant IBS complain of frequent bowel movements, loose or watery stools and urgency of bowel movement [1, 2]. The latter is a major concern of patients with diarrheapredominant IBS.
In this era of managed care, many of the expensive and sophisticated tests available are generally not needed for patients with positive symptom criteria and no features suggestive of organic disease [3]. However, alarm symptoms suggestive of organic disease, which include blood per rectum, anemia, loss of weight or appetite, fever, nocturnal diarrhea and a positive family history of Crohn's or celiac disease must be sought. Minimal diagnostic tests have been advocated in the initial diagnostic approach to patients with suspected IBS to reasonably exclude structural and inflammatory lesions and to convince us and the patient of the diagnosis of IBS [84]. The basic tests that should be obtained include complete blood count, erythrocyte sedimentation rate, blood chemistries, thyroid panel and stool for occult blood (three times) as well as ova and parasites [1]. Visualization of the colon with flexible sigmoidoscopy is indicated in those less than 50 years old with no alarm symptoms and without a family history of colon cancer. Patients over 50 years of age or with a family history of colon cancer or polyps or with alarm symptoms should have complete colonoscopy. This investigation has been shown to have a yield of approximately $1 \%$, finding structural lesions such as polyps, colon cancer and colitis [85].

Follow-up studies extending up to 9 years after the diagnosis of IBS, using symptom criteria and limited diagnostic evaluations in the appropriate patient subgroups, have found that fewer than $5 \%$ of patients had other explanations for their symptoms [77, 86]. An extensive workup is usually not indicated in patients with typical IBS symptoms, and further investigation only serves to undermine the patient's confidence in the diagnosis and the physician [87]. More detailed investigations are indicated in patients older than 50 years and patients who have severe or disabling symptoms, experience a change of clinical course or develop alarm symptoms such as weight loss or heme-positive stool.

\section{Management}

A meta-analytic review in 1988 by Klein [88] showed that the majority of trials of therapeutic agents in IBS were flawed, and that none of the agents considered in his review could be regarded as effective in the treatment of IBS. However, there has been a great deal of improvement in the design of more recent trials, including the improved characterization of patient subgroups and better trial design of appropriately powered studies with definable, clinically relevant end points. These trials have directed 
Fig. 1. Algorithm of diagnostic and therapeutic approach to IBS based on predominant symptom subgrouping. ESR = Erythrocyte sedimentation rate; $\mathrm{TSH}=$ thyroid stimulating hormone.

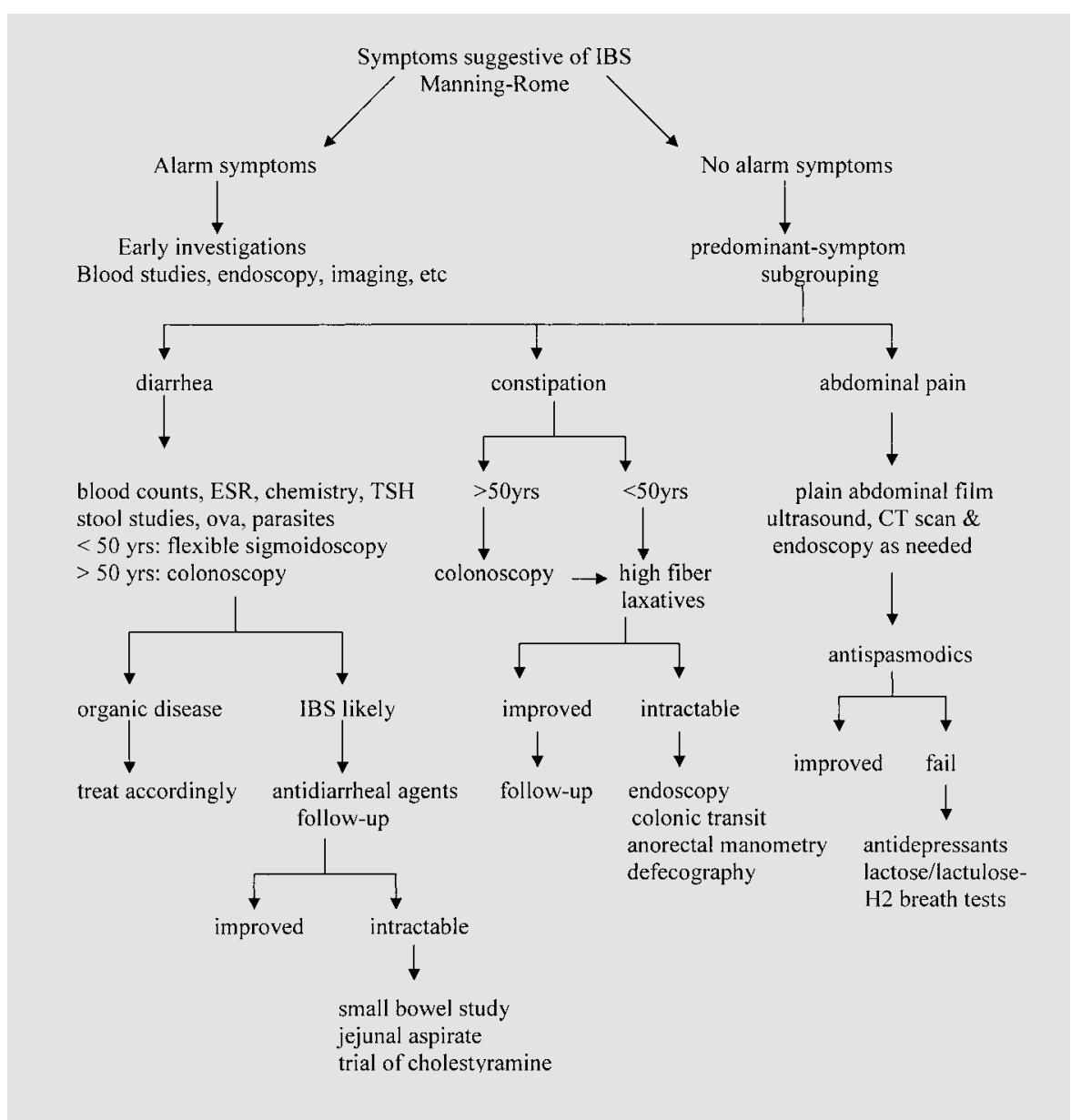

our therapeutic approach and were recently reviewed by Jailwala et al. [89]. The American Gastrointestinal Association has suggested strategies for the diagnosis and management of patients with IBS [3]. With a greater understanding of the brain-gut axis and the development of novel pharmacologic agents, our future management of IBS patients should improve [89].

The therapeutic goals in IBS must focus on the overall well-being of the patient, including abdominal symptoms, the accompanying nonbowel symptoms and the high prevalence of affective disorders [6]. It is also important to establish an effective physician-patient relationship and to reassure the patient once the diagnosis of IBS is made $[3,11]$. Reassurance, as we know, has therapeutic value and should decrease the patient's concerns after an adequate and generally conservative diagnostic evaluation [3]. Symptom-based subgrouping of patients is helpful in selecting the best management approach for an individual patient (fig. 1).

Pathogenesis and Management of Irritable Bowel Syndrome

\section{Constipation-Predominant IBS}

Increased dietary fiber or psyllium products are frequently recommended for patients with constipation-predominant IBS, even though as a group, these patients do not consume less dietary fiber than control subjects [3]. Most studies report a significant improvement in constipation if sufficient quantities of fiber (20-30 g/day) are consumed [90]. The use of fiber decreases the whole gut transit time, which may alleviate constipation by decreasing intracolonic pressure. Decreased intracolonic pressure could reduce pain and dilute bile salts, which in turn could indirectly reduce colonic contractile activity [91]. Double-blind placebo-controlled studies have demonstrated that increased bulk with fiber or roughage is likely to be effective in most patients with predominant constipation [90, 92]. Long-term fiber supplementation had an equivocal benefit in a group of 14 patients with IBS followed for 2-3 years [93]. Because of the uncertain results, 
it has been suggested that a formal reappraisal of the effectiveness of fiber in IBS be performed [94]. The major side effect of fiber is abdominal bloating and increased flatulence; therefore, it should be used cautiously and in low doses.

Cisapride, a prokinetic drug with 5-HT4 agonist and 5-HT3 antagonist action that facilitates acetylcholine release from the myenteric plexus, has been reported to be helpful in constipation-predominant IBS [95]. Two other high-quality trials, however, showed that cisapride was not superior to placebo in the treatment of constipation and abdominal discomfort in IBS [96, 97]. It has a role as a promotility agent in the upper gastrointestinal tract. However, in July 2000, the manufacturer withdrew it from the US market because of cardiac side effects. An osmotic laxative such as milk of magnesia or lactulose or a stool softener may be added to the therapeutic regimen if a bulk laxative alone is insufficient [11].

Two novel serotonin agents with promotility actions are currently under development: tegaserod (a 5-HT4 receptor partial agonist) for IBS and prucalopride (a 5HT4 receptor full agonist) for chronic constipation. Tegaserod produces a gastrointestinal stimulatory effect partially by facilitation of cholinergic transmission [98]. Animal studies evaluating the effects of tegaserod showed stimulatory effects on motor activity throughout the gastrointestinal tract in various species $[34,98]$. The effect of tegaserod on gastric, small bowel and colonic transit was studied in 24 patients with constipation-predominant IBS. It was found to facilitate about a $20 \%$ acceleration of proximal colonic transit [98]. In a randomized, doubleblind, parallel-group study, 881 patients with constipation-predominant IBS were given tegaserod (4 or $12 \mathrm{mg}$ ) or placebo during 12 weeks of treatment [99]. The subject's global assessment of relief measures was obtained weekly, using a 5-point ordinal scale (completely, considerably, somewhat, unchanged or worse) as the primary end point. The subject's global assessment of relief measure encompassed relief of abdominal pain, bowel movement and overall well-being. At week 1, a significant increase in relief was apparent among patients taking tegaserod $(12 \mathrm{mg} /$ day $)$ compared with their counterparts on placebo. This effect was sustained through the $3 \mathrm{rd}$ month of the study [99].

Prucalopride has been shown to induce high-amplitude propagating contractions in a dose-dependent manner, accelerating colonic transit and causing mass movement in healthy volunteers [100]. A small study showed that prucalopride increased the percentage of loose stools and the overall number of stools per week in healthy vol- unteers [101]. Second-generation 5-HT4 receptor agonists like tegaserod and prucalopride seem to be devoid of the QT-prolonging effects observed in some clinical circumstances with cisapride [102]. In a double-blind parallel study, prucalopride accelerated gastric emptying, small bowel transit, overall colonic transit and ascending colon emptying in patients with functional constipation [103]. However, the effect of prucalopride on constipation was not addressed in this 1-week study, and certainly more studies would be needed to evaluate the utility of this novel agent in the treatment of constipation-predominant IBS.

\section{Diarrhea-Predominant IBS}

In patients with predominant diarrhea, a detailed history may identify factors, e.g. intake of drugs or dietary factors such as sorbitol, furctose or lactose, that may be aggravating or causing symptoms. There is no proven role for dietary fiber in treating diarrhea associated with IBS. A controlled crossover study of corn fiber and placebo showed improvement in stool frequency, stool consistency, pain severity and total symptom score in both the fiber and the placebo group [104]. In IBS patients with predominant diarrhea, small bowel and proximal colonic transit is increased [105]. Loperamide (Imodium), a synthetic opioid, decreases intestinal transit, enhances intestinal water and ion absorption and increases anal sphincter tone at rest [106]. These effects seem to explain the improvement in diarrhea, urgency and fecal soiling observed in patients with IBS who use loperamide [107]. Loperamide is generally preferred to other opiates such as diphenoxylate (Lomotil), codeine or other narcotics. Their mechanism of action is mediated primarily by peripheral mu receptors in the gastrointestinal tract which reduce gut motility and secretions, allowing for greater fluid absorption and improved stool consistency [21]. They may also benefit postprandial urgency that is associated with a prominent colonic response to eating [106]. Several clinical studies have demonstrated this ability of opioids to improve diarrhea and fecal urgency associated with IBS [21]. Lavo et al. [108] found that loperamide improved stool consistency, abdominal pain, fecal urgency and overall well-being.

Recently, alosetron, a 5-HT3 receptor antagonist, became available for the treatment of diarrhea-predominant IBS in female patients. It may act at multiple levels by inhibiting visceral sensitivity, increasing compliance and inhibiting excitatory 5-HT3 receptors located on ascend- 
ing and descending neuronal pathways involved in peristalsis. A large multinational study of alosetron showed a $27 \%$ absolute difference in the frequency of global improvement and significant improvement in stool frequency, consistency and percentage of days with urgency among females compared to placebo [109]. In another study, alosetron was compared to mebeverine (smooth muscle antagonist) in a multicenter randomized doubleblind study in nonconstipated females with IBS and was found to be effective in controlling both abdominal pain and bowel dysfunction [110]. Moreover, it was significantly more effective than mebeverine in the control of multiple IBS symptoms in this population [110]. Soon after its approval for treating female patients with diarrhea-predominant IBS, alosetron was voluntarily withdrawn from the market in November 2000 because of its association with ischemic colitis [111]. The reported cases resolved after several days to weeks without sequelae. Whether this is a side effect confined to alosetron or represents a class effect common to all 5-HT3 antagonists needs to be determined. Other drugs in this class, for example cilanteron, are being studied [112].

\section{Abdominal Pain}

Pain is the dominant symptom of IBS, and along with bloating, is the symptom least amenable to drug treatment. Although many patients may attribute specific food substances to their symptoms, by and large, the type of food does not generally contribute to symptoms [3]. However, some dietary substances may aggravate symptoms in some individuals. These might include fatty foods, beans, alcohol, caffeine, lactose and in some cases excess fiber [3, 11]. The patients should avoid the possible inciting dietary factors and monitor the response. However, care should be taken to avoid an unnecessarily restrictive diet [3]. While dietary fiber has a role in treating constipation, its value in the relief of abdominal pain is not established. Fiber intake, however, has been shown to alleviate pain in children with idiopathic chronic abdominal pain [113]. In a double-blind placebo-controlled crossover trial of bran therapy in IBS patients, both the treatment and the study groups experienced an improvement in symptoms, especially abdominal pain. The beneficial effect was due to a placebo response, which was independent of the increase in stool weight [114].

Only smooth muscle relaxants have been found to consistently decrease abdominal pain in IBS patients [89]. There are a few studies that have individually showed sig- nificant benefit, and a recent meta-analysis of 26 studies by Poynard et al. [115] concluded that, as a therapeutic class, smooth muscle relaxants or antispasmodics were significantly more effective than placebo in producing global improvement (62 vs. $35 \%$ placebo improvement) and abdominal pain (64 vs. $45 \%$ placebo improvement) (both $\mathrm{p}<0.01$ ). Dicyclomine is widely used in the United States, although its efficacy is supported by only one trial [116]. In this trial, dicyclomine was associated with global improvement and an improvement in abdominal pain and constipation. Most treated patients experienced anticholinergic side effects (69\% compared with $16 \%$ of controls). Four smooth muscle relaxants (cimetropium, pinaverium, otilonium and trimebutine) have consistently been shown to be efficacious in high-quality trials [89], and the lower frequency of systemic anticholinergic side effects with selective antimuscarinic agents such as cimetropium and pinaverium is clearly desirable. None of these agents is currently approved for the treatment of IBS in the United States. In clinical practice, antispasmodics are best used on an as-needed basis for acute attacks of pain, distension or bloating, as they may become less effective with long-term use [112].

5-HT3 antagonists might also be useful in the relief of abdominal pain because they reduce postprandial colonic tone and seem to reduce colonic sensitivity. In a phase II study, the 5-HT3 receptor antagonist alosetron was significantly better than the placebo in relieving pain, reducing bowel frequency and urgency and improving stool consistency, although significant differences between alosetron and placebo were only apparent in women [117].

\section{Psychologic/Psychotropic Treatment}

Although less readily available, psychological treatment may help in the managment of selected patients with IBS. Talley et al. [118], by performing a systematic review of psychological treatment of IBS, concluded that the efficacy of psychologic treatment has not been established because of methodologic inadequacy. Although 8 studies reported psychologic treatment to be superior to control therapy, 5 failed to show any significant effect [118]. Despite methodological limitations, the available data support the value of psychological treatment (1) in reducing anxiety and other psychological symptoms, and (2) in a subset of patients whose symptoms, particularly intermittent abdominal pain and diarrhea, are moderate to severe and are associated with psychological distress and impaired quality of life $[3,119,120]$. Psychological 
treatments that have been studied in relation to IBS include cognitive-behavioral treatment, hypnosis, psychodynamic or interpersonal psychotherapy and stress management.

Antidepressants, either the tricyclic agents or selective serotonin reuptake inhibitors, are now frequently used to treat patients with IBS, particularly those with severe or refractory symptoms and impaired daily function. Their previous use in IBS patients was based on the fact that a high proportion of these patients reported depressive symptoms [121]. However, it is now recognized that the antidepressants have neuromodulatory and analgesic properties independent of their psychotropic effect [122]. A recent review of 7 trials that evaluated tricyclic agents in IBS showed them to be beneficial. Global improvement was reported in 5 trials, 3 of which did not measure improvement in individual symptoms. Four of the 7 trials reported improvement in abdominal pain and diarrhea but none reported any difference in constipation [89]. Jackson et al. [123] performed a meta-analysis of 11 randomized controlled trials on the use of antidepressants for the treatment of patients with functional gastrointestinal disorders. Eight of the 11 studies focused exclusively on IBS, 2 on nonulcer dyspepsia and 1 included both IBS and nonulcer dyspepsia. Nine of the 11 trials studied tricyclic antidepressants and 2 trials used the antiserotonin agent mianserin. Five tricyclic antidepressants were studied: amitriptyline in 3 studies, tramimpramine in 3, desipramine in 2 and doxepin in 1 . No study made a direct comparison of antidepressants with other management options in IBS. The analysis suggests that antidepressants appear to be effective in the treatment of functional gastrointestinal disorders and that the magnitude of the benefit is clinically important. Patients treated with antidepressants were substantially more likely to improve, with about 3 patients needing treatment to improve 1 patient's symptoms. Since the studies reviewed used antidepressant doses that are generally subtherapeutic compared with what is needed for treating depression, it seems unlikely that the benefit is due entirely to the antidepressant properties of these drugs [123]. Further, since these studies did not control for psychological effects, it is not possible to know if the benefit received was independent of the antidepressants' effect on depression. There are no published controlled studies on the use of the new selective serotonin reuptake inhibitor antidepressants in patients with IBS. However, recent data indicate that they can be useful in patients with other chronic pain conditions regardless of a diagnosis of depression [124].

\section{Conclusion}

Our knowledge about different aspects of IBS continues to expand as numerous studies are published every month. We can be relatively confident in its presence whenever our patients fit the diagnostic criteria of IBS, and this will help us to avoid unnecessary investigations. Keeping the red flags in mind will direct us to selectively work up those patients who most likely have another organic disease. Understanding the role of the new concept of brain-gut interactions in the pathogenesis of IBS will continue to stimulate further research to develop newer agents that may affect the system at different levels. It is important to realize that symptoms of IBS may be influenced at different levels: at the viscus itself, at the spinal cord centers and higher in the brain. Further research in the field of gastrointestinal hormones, neurotransmitters and receptors is expected to further enrich our knowledge about IBS. A total therapeutic approach directed at abdominal symptoms, associated syndromes such as fibromyalgia and psychological aspects should be adopted in the management of IBS patients. 


\section{References}

1 Schmulson MW, Chang L: Diagnostic approach to the patient with irritable bowel syndrome. Am J Med 1999; 107:20S-26S.

2 Talley NF: Irritable bowel syndrome: Disease definition and symptom description. Eur J Surg Suppl 1998;583:24-28.

3 Drossman DA, Whitehead WE, Camilleri M: Irritable bowel syndrome - a technical review for practice guideline development. Gastroenterology 1997;112:2120-2137.

4 Hammer J, Talley NJ: Diagnostic criteria for the irritable bowel syndrome. Am J Med 1999; 107:5S-11S.

5 Drossman DA: The functional gastrointestinal disorders and the Rome II process. Gut 1999; 45(suppl 2):II1-II5.

6 Farthing MJ: Irritable bowel syndrome: New pharmaceutical approaches to treatment. Baillières Best Pract Res Clin Gastroenterol 1999; 13:461-471

7 Drossman DA, Li Z, Andruzzi E, Temple RD, Talley NJ, Thompson WG, Whitehead WE, Janssens J: U.S. householder survey of functional gastrointestinal disorders: Prevalence, sociodemography, and health impact. Dig Dis Sci 1993;38:1569-1580.

8 Talley NJ, Zinsmeister AR, Van Dyke C, Melton LJ 3rd: Epidemiology of colonic symptoms and the irritable bowel syndrome. Gastroenterology 1991;101:927-934.

9 Longstreth GF, Wolde-Tsadik G: Irritable bowel-type symptoms in HMO examinees: Prevalence, demographics and clinical correlates. Dig Dis Sci 1993;38:1581-1589.

10 Taub E, Cuevas JL, Cook EW, Crowell M, Whitehead WE: Irritable bowel syndrome defined by factor analysis: Gender and race comparisons. Dig Dis Sci 1995;40:2647-2655.

11 Camilleri M, Choi MG: Review article: Irritable bowel syndrome. Aliment Pharmacol Ther 1997;11:3-15.

12 Schlemper RJ, Van der Werf SDJ, Vandenbroucke JP, Biemond I, Lamers CB: Peptic ulcer, non-ulcer dyspepsia and irritable bowel syndrome in the Netherlands and Japan. Scand J Gastroenterol suppl 1993;200:33-41.

13 Bi-zhen W, Qi-ying P: Functional bowel disorders in apparently healthy Chinese people. Chin J Epidemiol 1988;9:345-349.

14 Olubuyide IO, Olawuyi F, Fasanmade AA: A study of irritable bowel syndrome diagnosed by Manning criteria in an African population. Dig Dis Sci 1995;40:983-985.

15 Talley NJ, Zinsmeister AR, Melton LJ 3rd: Irritable bowel syndrome in a community: Symptom subgroups, risk factors and health care utilization. Am J Epidemiol 1995; 142:7683.

16 Everhart JE, Renault PF: Irritable bowel syndrome in office based practice in the United States. Gastroenterology 1991;100:998-1005.

17 Sandler RS: Epidemiology of irritable bowel syndrome in the United States. Gastroenterology 1990;99:409-415.
18 Mitchell CM, Drossman DA: Survey of the AGA membership relating to patients with functional gastrointestinal disorders. Gastroenterology 1987;92:1282-1284.

19 Longstreth GF: Irritable bowel syndrome: A multibillion-dollar problem. Gastroenterology 1995;109:2029-2031.

20 Talley NJ, Gabriel SE, Harmsen WS, Zinsmeister AR, Evans RW: Medical costs in community subjects with irritable bowel syndrome. Gastroenterology 1995;109:1736-1741.

21 Mayer EA, Chang L, Lembo T: Brain-gut interactions: Implications for newer therapy. Eur $\mathrm{J}$ Surg Suppl 1998;582:50-55.

22 Wood JD, Alpers DH, Andrews PL: Fundamentals of neurogastroenterology. Gut 1999; 45(suppl 2):II6-II16.

23 Kellow JE, Delvaux M, Azpiroz F, Camilleri M, Quigley EM, Thompson DG: Principles of applied neurogastroenterology: Physiology/ motility-sensation. Gut 1999;45(suppl 2):II17II24.

24 Santos J, Saperas E, Mourelle M, Antolin M, Malagelada JR: Regulation of intestinal mast cells and luminal protein release by cerebral thyrotopin-releasing hormone in rats. Gastroenterology 1996;111:1465-1473.

25 Gue M, Del Rio-Lacheze C, Eutamene H, Theodorou V, Fioramonti J, Bueno L: Stressinduced visceral hypersensitivity to rectal distension in rats: Role of CRF and mast cells. Neurogastroenterol Motil 1997;9:271-279.

26 Coelho AM, Fioramonti J, Bueno L: Mast cell degranulation induces delayed rectal allodynia in rats: Role of histamine and 5-HT. Dig Dis Sci 1998;43:727-737.

27 Rodriguez LA, Ruigomez A: Increased risk of irritable bowel syndrome after bacterial gastroenteritis: Cohort study. BMJ 1999;318:565566.

28 Gwee KA, Leong YL, Graham C, McKendrick MW, Collins SM, Walters SJ, Underwood JE, Read NW: The role of psychological and biological factors in postinfective gut dysfunction. Gut 1999;44:400-406.

29 Gershon MD: Review article: Roles played by 5-hydroxytryptamine in the physiology of the bowel. Aliment Pharmacol Ther 1999; 13(suppl 12):15-30.

30 Kim DY, Camilleri M: Serotonin: A mediator of the brain-gut connection. Am J Gastroenterol 2000;95:2698-2709.

31 Goyal RK, Hirano I: The enteric nervous system. N Engl J Med 1996;334:1106-1115.

32 Sanger GJ: Different pathophysiological functions of the 5-HT4 and 5-HT3 receptors in small and large intestine. Curr Res Serotonin 1998;3:99-104.

33 Sanger GJ: Therapeutic applications of 5-HT4 receptor agonists and antagonists; in Eglen RM (ed): 5HT4 Receptors in the Brain and Periphery. Springer/Landes, 1998, pp 213-226.

34 Grider JR, Foxx-Orenstein AE, Jin JG: 5Hydroxytryptamine-4 receptor agonists initiate the peristaltic reflex in human, rat and guinea pig intestine. Gastroenterology 1998; 115:370-380.
35 Sanger GJ: 5-Hydroxytryptamine and functional bowel disorders. Neurogastroenterol Motil 1996;8:319-331.

36 Camilleri M, Neri M: Motility disorders and stress. Dig Dis Sci 1989;34:1777-1786.

37 Whitehead WE, Engel BT, Schuster MM: Irritable bowel syndrome: Physiological and psychological differences between diarrhea-predominant and constipation-predominant patients. Dig Dis Sci 1980;25:404-413.

38 Bazzocchi G, Ellis J, Villanueva-Meyer J, Jing J, Reddy SN, Mena I, Snape WJ Jr: Postprandial colonic transit and motor activity in chronic constipation. Gastroenterology 1990; 98:686-693.

39 Cann PA, Read NW, Brown C, Hobson N, Holdsworth CG: Irritable bowel syndrome: Relationship of disorders in the transit of a single solid meal to symptom patterns. Gut $1983 ; 24$ : 405-411.

40 Welgan P, Meshkinpour H, Beeler M: Effect of anger on colon motor and myoelectric activity in irritable bowel syndrome. Gastroenterology 1988;94:1150-1156.

41 Valentino RJ, Miselis RR, Pavcovich LA: Pontine regulation of pelvic viscera: Pharmacological target for pelvic visceral dysfunctions. Trends Pharmacol Sci 1999;20:253-260.

42 Kellow JE, Eckersley CM, Jones MP: Enhanced perception of physiological intestinal motility in the irritable bowel syndrome. Gastroenterology 1991;101:1621-1627.

43 Whitehead WE, Holtkotter B, Enck P, Hoelz R, Holmes KD, Anthony J, Shabsin HS, Schuster MM: Tolerance for rectosigmoid distension in irritable bowel syndrome. Gastroenterology 1990;98:1187-1192.

44 Prior A, Maxton DG, Whorwell JP. Anorectal manometry in irritable bowel syndrome: Differences between diarrhea and constipation predominant subjects. Gut 1990;31:458-462.

45 Mayer EA, Gebhart GF: Basic and clinical aspects of visceral hyperalgesia. Gastroenterology 1994;107:271-293.

46 Mertz H, Naliboff B, Munakata J, Niazi N, Mayer EA: Altered rectal perception is a biological marker of patients with irritable bowel syndrome? Gastroenterology 1995;109:40-52.

47 Whitehead WE, Palsson OS: Is rectal pain sensitivity a biological marker for irritable bowel syndrome? Psychological influences on pain perception. Gastroenterology 1998;115:12631271.

48 Swarbrick ET, Hegarty JE, Bat L, Williams CB, Dawson AM: Site of pain from the irritable bowel. Lancet 1980;ii:443-446.

49 Kozlowski CM, Green A, Grundy D, Boissonade FM, Bountra C: The 5-HT3 receptor antagonist alosetron inhibits the colorectal distension-induced depressor response and spinal cfos expression in the anaesthetised rat. Gut 2000;46:474-480.

50 Junien JL, Riviere P: Review article: The hypersensitive gut - peripheral kappa agonists as a new pharmacological approach. Aliment Pharmacol Ther 1995;9:117-126. 
51 Delvaux M, Louvel D, Lagier E, Scherrer B, Abitbol JL, Frexinos J: The kappa agonist fedotozine relieves hypersensitivity to colonic distension in patients with irritable bowel syndrome. Gastroenterology 1999;116:38-45.

52 Silverman DH, Munakata JA, Ennes H, Mandelkern MA, Hoh CK, Mayer EA: Regional cerebral activity in normal and pathological perception of visceral pain. Gastroenterology 1997;112:64-72.

53 Mayer EA, Fass R, Fullerton S: Intestinal and extraintestinal symptoms in functional gastrointestinal disorders. Eur J Surg Suppl 1998; 583:29-31.

54 Jaenig W, Morrison JF: Progress in Brain Research. 7: Functinal Properties of Spinal Visceral Afferents Supplying Abdominal and Pelvic Organs, with Special Emphasis on Visceral Nociception. Amsterdam, Elsevier Science, 1995, p 87.

55 Drossman DA, Creed FH, Olden KW, Svedlund J, Toner BB, Whitehead WE: Psychosocial aspects of the functional gastrointestinal disorders. Gut 1999;45(suppl 2):II25-II30.

56 Whitehead WE, Crowell MD, Robinson JC, Heller BR, Schuster MM: Effects of stressful life events on bowel symptoms: Subjects with irritable bowel syndrome compared with subjects without bowel dysfunction. Gut 1992;33 825-830.

57 Creed F, Craig T, Farmer R: Functional abdominal pain, psychiatric illness, and life events. Gut 1988;29:235-242.

58 Drossman DA, Sandler RS, McKee DC, Lovitz AJ: Bowel patterns among subjects not seeking health care. Use of a questionnaire to identify a population with bowel dysfunction. Gastroenterology 1982;83:529-534.

59 Talley NJ, Howell S, Poulton R: The irritable bowel syndrome and psychiatric disorder in the community: Is there a link? Am J Gastroenterol 2001:96:1072-1079.

60 Whitehead WE, Bosmajian L, Zonderman AB, Costa PT Jr, Schuster MM: Symptoms of psychological distress associated with irritable bowel syndrome. Comparison of community and medical clinic samples. Gastroenterology 1988;95:709-714.

61 Whitehead WE: Psychosocial aspects of functional gastrointestinal disorders. Gastroenterol Clin North Am 1996;25:21-34.

62 Drossman DA, Talley NJ, Leserman J, Olden KW, Barreiro MA: Sexual and physical abuse and gastrointestinal illness. Review and recommendations. Ann Intern Med 1995;123:782794.

63 Drossman DA, Li Z, Leserman J, Toomey TC, $\mathrm{Hu}$ YJ: Health status by gastrointestinal diagnosis and abuse history. Gastroenterology 1996;110:999-1007.

64 Manning AP, Thompson WG, Heaton KW, Morris AF: Towards positive diagnosis of the irritable bowel. Br Med J 1978;2:653-654.

65 Thompson WG: Gastrointestinal symptoms in the irritable bowel syndrome compared with peptic ulcer and inflammatory bowel disease. Gut 1984;25:1089-1092.
66 Talley NJ, Phillips SF, Melton LJ, Mulvihill C, Wiltgen C, Zinsmeister AR: Diagnostic value of the Manning criteria in irritable bowel syndrome. Gut 1990;31:77-81.

67 Rao KP, Gupta S, Jain AK, Agrawal AK, Gupta JP: Evaluation of Manning criteria in the diagnosis of irritable bowel syndrome. J Assoc Physicians India 1993;41:357-358.

68 Jeong H, Lee HR, Yoo BC, Park SM: Manning criteria in irritable bowel syndrome: Its diagnostic significance. Korean J Intern Med 1993; 8:34-39.

69 Smith RC, Greenbaum DS, Vancouver JB, Henry RC, Reinhart MA, Greenbaum RB, Dean HA, Mayle JE: Gender differences in Manning criteria in the irritable bowel syndrome. Gastroenterology 1991;100:591-595.

70 Kruis W, Thieme C, Weinzierl M, Schussler P, Holl J, Paulus W: A diagnostic score for the irritable bowel syndrome. Its value in the exclusion of organic disease. Gastroenterology 1984; 87:1-7.

71 Talley NJ: Irritable bowel syndrome: Definition, diagnosis and epidemiology. Ballières Best Pract Res Clin Gastroenterol 1999;13: 371-384.

72 Frigerio G, Beretta A, Orsenigo G, Tadeo G, Imperiali G, Minoli G: Irritable bowel syndrome. Still far from a positive diagnosis. Dig Dis Sci 1992;37:164-167.

73 Dogan UB, Unal S: Kruis scoring system and Manning's criteria in diagnosis of irritable bowel syndrome: Is it better to use combined? Acata Gastroenterol Belg 1996;59:225-228.

74 Thompson WG, Creed F, Drossman DA, Heaton KW, Mazzacca G: Functional bowel disease and functional abdominal pain. Gastroenterol Int 1992;5:75-91.

75 Thompson WG, Longstreth GF, Drossman DA, Heaton KW, Irvine EJ, Muller-Lissner SA: Functional bowel disorders and functional abdominal pain. Gut 1999;45(suppl 2):II43II47.

76 Talley NJ, Holtman J, Zinsmeister AR, Jones $\mathrm{M}$ : Gastrointestinal symptom cluster into distinct upper and lower grouping consistent with the Rome classification: A three country population-based study. Gastroenterology 1997; 112:A835.

77 Vanner SJ, Depew WT, Paterson WG, DaCosta LR, Groll AG, Simon JB, Djurfeldt M: Predictive value of the Rome criteria for diagnosing the irritable bowel syndrome. Am J Gastroenterol 1999;94:2912-2917.

78 Whorwell PJ, McCallum M, Creed FH, Roberts CT: Non-colonic features of irritable bowel syndrome. Gut 1986;27:37-40.

79 Talley NJ, Phillips SF, Bruce B, Zinsmeister AR, Wiltgen C, Metton LJ: Multisystem complaints in patients with the irritable bowel syndrome and functional dyspepsia. Eur J Gastroenterol Hepatol 1991;3:71-77.

80 Wessely S, Nimnuan C, Sharpe M: Functional somatic syndromes: One or many? Lancet 1999;354:936-939.

81 Kay L, Jorgensen T: Redefining abdominal syndromes. Results of a population-based study. Scand J Gastroenterol 1996;31:469475 .
82 Talley NJ, Boyce P, Jones M: Identification of distinct upper and lower gastrointestinal symptom grouping in an urban population. Gut 1998;42:690-695.

83 Whitehead WE: Patient subgroups in irritable bowel syndrome that can be defined by symptom evaluation and physical examination. Am J Med 1999;107:33S-40S

84 Camilleri M, Prather CM: The irritable bowel syndrome: Mechanisms and a practical approach to management. Ann Intern Med 1992; 116:1001-1008.

85 McIntosh DG, Thompson GW, Patel DG, Barr $\mathrm{R}$, Guindi $\mathrm{M}$ : Is rectal biopsy necessary in irritable bowel syndrome? Am J Gastroenterol 1992;87:1407-1409.

86 Svendsen JH, Munck LK, Andersen JR: Irritable bowel syndrome: Prognosis and diagnostic safety. A 5-year follow-up study. Scand J Gastroenterol 1985;20:415-418.

87 Thompson GW: Functional bowel disorders and functional abdominal pain; in Drossman DA, Richter J, Talley NJ, Thompson GW, Corazziari E, Whitehead WE (eds): The Functional Gastrointestinal Disorders. Boston, Little, Brown, 1994, pp 115-174.

88 Klein KB: Controlled treatment trials in the irritable bowel syndrome: A critique. Gastroenterology 1988;95:232-241.

89 Jailwala J, Imperiale TF, Kroenke K: Pharmacologic treatment of the irritable bowel syndrome: A systematic review of randomized controlled trials. Ann Intern Med 2000;133: 136-147.

90 Cann PA, Read NW, Holdsworth CD: What is the benefit of coarse wheat bran in patients with irritable bowel syndrome? Gut 1984;25: 168-173.

91 Mueller-Lissner SA: Effect of wheat bran on weight of stool and gastrointestinal transit time: A meta-analysis. Br Med J 1988;296: 615-617.

92 Prior A, Whorwell PJ: Double-blind study of ispaghula in irritable bowel syndrome. Gut 1987;28:1510-1513.

93 Hillman LC, Stace NH, Pomare EW: Irritable bowel patients and their long-term response to a high-fiber diet. Am J Gastroenterol 1984;79: $1-7$.

94 Francis CY, Whorwell PJ: Bran and irritable bowel syndrome: Time for reappraisal. Lancet 1994;334:39-40.

95 Van Outryve M, Milo R, Toussaint J, Van Eeghem P: 'Prokinetic' treatment of constipation-predominant irritable bowel syndrome: A placebo-controlled study of cisapride. J Clin Gastroenterol 1991;13:49-57.

96 Schutze K, Brandstatter G, Dragosics B, Judmaier G, Hentschel E: Double-blind study of the effect of cisapride on constipation and abdominal discomfort as components of the irritable bowel syndrome. Aliment Pharmacol Ther 1997:11:387-394.

97 Farup PG, Hovdenak N, Wetterhus S, Lango OJ, Hovde O, Trondstad R: The symptomatic effect of cisapride in patients with irritable bowel syndrome and constipation. Scand J Gastroenterol 1998;33:128-131. 
98 Prather CM, Camilleri M, Zinsmeister AR, Mckinzie S, Thomforde G: Tegaserod accelerates ororectal transit in patients with constipation-predominant irritable bowel syndrome. Gastroenterology 2000;118:463-468.

99 Mueller-Lissner S, Fumagalli I, Bardhan K: Tegaserod, a 5HT4 receptor partial agonist relieves key sympotms of irritable bowel syndrome (IBS). Gastroenterology 2000;118 (4 suppl 2):A175.

100 Emmanuel AV, Kamm MA, Roy AJ, Antonelli K: Effect of a novel prokinetic drug, R093877, on gestrointestinal transit in healthy volunteers. Gut 1998;42:511-516.

101 Poen AC, Felt Bersma RJ, Van Dongen PA, Meuwissen SG: Effect of pucalopride, a new enterokinetic agent, on gastrointestinal transit and anorectal function in healthy volunteers. Aliment Pharmacol Ther 1999;13: 1493-1497.

102 De Ponti F, Tonini M: Irritable bowel syndrome: New agents targeting serotonin receptor subtypes. Drugs 2001;61:317-332.

103 Bouras EP, Camilleri M, Burton DD, Thomforde G, McKinzie S, Zinsmeister AR: Prucalopride accelerates gastrointestinal and colonic transit in patients with constipation without a rectal evacuation disorder. Gastroenterology 2001;120:354-360.

104 Cook IJ, Irvine EJ, Campbell D, Shannon S, Reddy SN, Collins SM: Effect of dietary fiber on symptoms and rectosigmoid motility in patients with irritable bowel syndrome. Gastroenterology 1990;98:66-72.

105 Vassallo MJ, Camilleri M, Phillips SF, Steadman CJ, Hanson RB, Haddad AC: Colonic tone and motility in patients with irritable bowel syndrome. Mayo Clin Proc 1992;67: 725-731.

106 Camilleri M: Therapeutic approach to the patient with irritable bowel syndrome. Am J Med 1999; 107:27S-32S.
107 Cann PA, Read NW, Holdsworth CD, Barends D: Role of loperamide and placebo in management of irritable bowel syndrome. Dig Dis Sci 1984;29:239-247.

108 Lavo B, Stenstam M, Nielsen AL: Loperamide in treatment of irritable bowel syndrome a double-blind placebo-controlled study. Scand J Gastroenterol Suppl 1987;130:7780 .

109 Camilleri M, Mayer EA, Drossman DA, Heath A, Dukes GE, McSorley D, Kong S, Mangel AW, Northcutt AR: Improvement in pain and bowel function in female irritable bowel patients with alosetron, a 5-HT3 receptor antagonist. Aliment Pharmacol Ther 1999;13:1149-1159.

110 Jones RH, Holtmann G, Ridrigo L, Ehsanullah R, Crompton PM, Jacques LA, Mills JG: Alosetron relieves pain and improves bowel function compared with mebeverine in female nonconstipated irritable bowel syndrome patients. Aliment Pharmacol Ther 1999;13:1419-1427.

111 Friedel D, Thomas R, Fisher RS: Ischemic colitis during treatment with alosetron. Gastroenterology 2001;120:557-560.

112 Camilleri M: Management of the irritable bowel syndrome. Gastroenterology 2001;120: 652-668.

113 Feldman W, McGrath P, Hodgson C, Ritter $\mathrm{H}$, Shipman RT: The use of dietary fiber in the management of simple, childhood idiopathic recurrent abdominal pain: Results in a prospective double-blind randomized controlled trial. Am J Dis Child 1985;138:12161218.

114 Lucey MR, Clark ML, Lowndes J, Dawson AM: Is bran efficacious in irritable bowel syndrome? A double blind, placebo-controlled crossover study. Gut 1987;28:221-225.

115 Poynard T, Naveau S, Mory B, Chaput JC: Meta-analysis of smooth muscle relaxants in the treatment of irritable bowel syndrome. Aliment Pharmacol Ther 1994;8:499-510.
116 Page JG, Dirnberger GM: Treatment of the irritable bowel syndrome with Bentyl (dicyclomine hydrochloride). J Clin Gastroenterol 1981;3:153-156.

117 Northcutt AR, Camilleri M, Mayer EA: Alosetron, a 5HT3 antagonist, is effective in the treatment of female irritable bowel syndrome patients (abstract). Gastroenterology 1998; 114:A839.

118 Talley NJ, Owen BK, Boyce P, Paterson K: Psychological treatment for irritable bowel syndrome: A critique of controlled treatment trials. Am J Gastroenterol 1996;2:277-283.

119 Guthrie E, Creed F, Dawson D, Tomenson B A controlled trial of psychological treatment for the irritable bowel syndrome. Gastroenterology 1991;100:450-457.

120 Drossman DA: Diagnosing and treating patients with refractory functional gastrointestinal disorders. Ann Intern Med 1995;123: 688-697.

121 Lancaster-Smith MJ, Prout BJ, Pinto T, Anderson JA, Schiff AA: Influence of drug treatment on the irritable bowel syndrome and its interaction with psychoneurotic morbidity. Acta Psychiatr Scand 1982;66:33-41.

122 Clouse RE: Antidepressants for functional gastrointestinal syndromes. Dig Dis Sci 1994; 39:2352-2363.

123 Jackson JL, O’Malley PG, Tomkins G, Balden E, Santoro J, Kroenke K: Treatment of functional gastrointestinal disorders with antidepressant medications: A meta-analysis. Am J Med 2000;108:65-72.

124 Finley PR: Selective serotonin reuptake inhibitors: Pharmacologic profiles and potential therapeutic distinctions. Ann Pharmacother 1994;28:1359-1369. 